\title{
InGaAs/GaAs multiquantum-well electroabsorption modulator with integrated waveguide
}

\author{
Utpal Das, Paul R. Berger, and Pallab K. Bhattacharya
}

Solid State Electronics Laboratory, Department of Electrical Engineering and Computer Science, The University of Michigan, Ann Arbor, Michigan 48109-2122

Received March 17, 1987; accepted June 25, 1987

\begin{abstract}
A monolithically integrated guided-wave modulator has been realized by using molecular-beam epitaxial regrowth and ion-milling techniques. The guiding and modulating regions consist, respectively, of In-doped GaAs and $\mathrm{GaAs} / \mathrm{In}_{0.34} \mathrm{Ga}_{0.66} \mathrm{As}$ strained-layer multiquantum wells. Modulation is achieved by field-enhanced electroabsorption in the multiquantum wells. The insertion loss of the modulator is $0.9 \mathrm{~dB}$, and the transmission loss in the guides is $\leq 1 \mathrm{~dB} / \mathrm{cm}$. The temporal response of similar GaAs/InGaAs as-grown photodiodes to pulsed laser excitation is characterized by a rise time of 115 psec.
\end{abstract}

For the development of monolithic optical integrated circuits based on III-V compound semiconductors, two components of particular interest are external modulators for junction lasers and spatial light modulators for computing applications. High-speed modulation in multiquantum-well (MQW) P-I-N diodes ${ }^{1}$ has been demonstrated in which the modulation is based on the quantum-confined Stark effect in these materials. In the P-I-N diode the light was incident and transmitted perpendicular to the layers. Wood et $a l .{ }^{2}$ have reported a leaky-waveguide modulator based on the same principle. However, the measured insertion loss of the device was $\sim 4 \mathrm{~dB}$ owing to a large $(\sim 3$ $\mathrm{dB}$ ) radiation loss. An efficient guided-wave modulator must meet two essential requirements: the guide must be low loss, and the insertion loss of the modulator should be small. In addition, depending on the particular application, a large modulation depth and/ or modulation speed may be required. We report in this Letter the fabrication and properties of a monolithically integrated guided-wave modulator that meets most of these requirements. The integrated device has an In-doped GaAs and an InGaAs/GaAs strained-layer superlattice (SLS) in the guiding and modulation regions, respectively, and is realized by molecular-beam epitaxial (MBE) regrowth and ionbeam milling techniques.

The integrated structures were grown on S-doped $n^{+}$GaAs substrates. First a $0.3-\mu \mathrm{m}$ GaAs buffer layer $\left(n=2 \times 10^{18} \mathrm{~cm}^{-3}\right)$ was grown, followed by the $2-\mu \mathrm{m}$ thick undoped $\mathrm{In}_{0.01} \mathrm{Ga}_{0.99} \mathrm{As}$ guiding layer. It was found by us that such lightly In-doped GaAs layers grown by MBE have a low density of defects and traps ${ }^{3}$ and can be used to realize extremely low-loss optical guides. ${ }^{4}$ The samples were then removed from the growth system, and $200 \mathrm{~nm}$ of $\mathrm{SiO}_{2}$ was deposited upon the epitaxial layers. Circular holes, $110 \mu \mathrm{m}$ in diameter, were then defined by photolithography and etching, first with buffered $\mathrm{HF}$ to remove the $\mathrm{SiO}_{2}$ layer and then with $1 \mathrm{H}_{3} \mathrm{PO}_{4}: 5 \mathrm{H}_{2} \mathrm{O}: 1 \mathrm{H}_{2} \mathrm{O}_{2}$ to remove the grown $\mathrm{GaAs}$ layers and approximately $0.8-1.0 \mu \mathrm{m}$ of the substrate. After a final etch of the exposed GaAs surface in $1 \mathrm{HCl}: 1 \mathrm{H}_{2} \mathrm{O}$, the samples were introduced into the growth chamber for regrowth of the modulator/detector structure. A schematic of this device is shown in Fig. 1(a) and is essentially a $p^{+}$ (InGaAs) $-i$ (InGaAs/GaAs MQW) $-n^{+}$(InGaAs) diode structure grown on suitable InGaAs buffer layers. The strained-layer MQW consists of 10-nm $\mathrm{In}_{0.34} \mathrm{Ga}_{0.66}$ As wells and $15-\mathrm{nm}$ GaAs barriers. These parameters were chosen so that the effective band gap of the MQW is close to and larger than the energy of the 1.15- $\mu \mathrm{m} \mathrm{He-Ne} \mathrm{test} \mathrm{source.} \mathrm{The} \mathrm{regrown} \mathrm{samples}$ were taken out, and the polycrystalline $\mathrm{GaAs}$ on the $\mathrm{SiO}_{2}$ layer and then the $\mathrm{SiO}_{2}$ layer itself were etched away. The photodiode and waveguide structures were then defined by a single ion-milling step through a $150-\mathrm{nm}$ Ti mask. Finally, appropriate ohmic contacts on the diode were evaporated and alloyed. A photomicrograph of a typical integrated device is shown in Fig. 1(b).

The single-mode guides are $8 \mu \mathrm{m}$ wide and $2 \mu \mathrm{m}$ thick. Propagation losses in these guides were independently measured by the cutback method, using a $1.15-\mu \mathrm{m} \mathrm{He}-\mathrm{Ne}$ laser. The guides were endfired on the cleaved edge with a focused $2-\mu \mathrm{m}$ spot. The output power was monitored for various guide lengths, and such data are shown in Fig. 2. Loss coefficients of $\leq 1.0 \mathrm{~dB} / \mathrm{cm}$ are measured in the $\mathrm{In}_{0.01} \mathrm{Ga}_{0.99} \mathrm{As}$ waveguides, which are among the lowest measured for III-V optical guides.5,6 The current-voltage characteristics of the modulators in the dark exhibit excellent rectifying characteristics, with a forward threshold voltage of $0.5 \mathrm{~V}$ and a reverse breakdown voltage of $16-20 \mathrm{~V}$. The leakage current at this bias is $10-15 \mu \mathrm{A}$, which is extremely low.

In order to understand the modulation characteristics of the MQW device, we measured the light transmission through the layers in a separate sample. Substrate removal was not necessary since the band gap of $\mathrm{GaAs}$ is larger than that of the InGaAs layers and MQW's grown on it. The sample was mounted with 


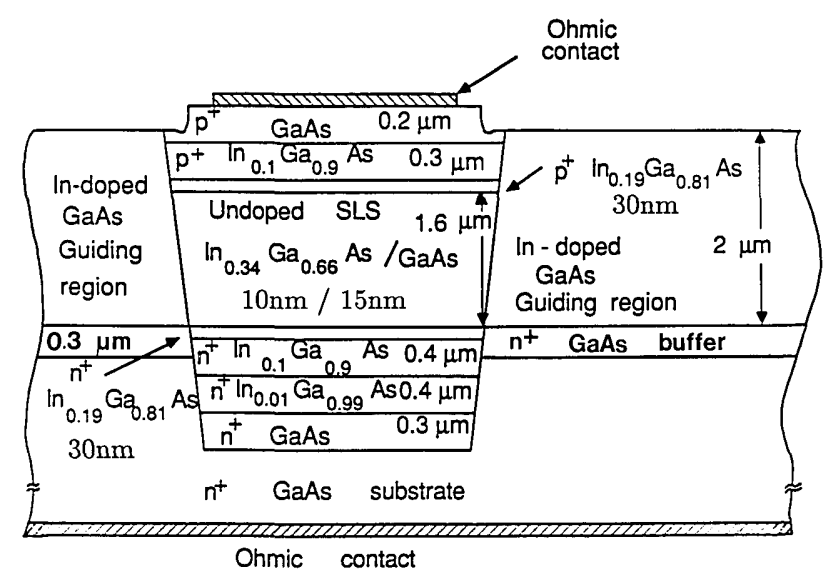

(a)

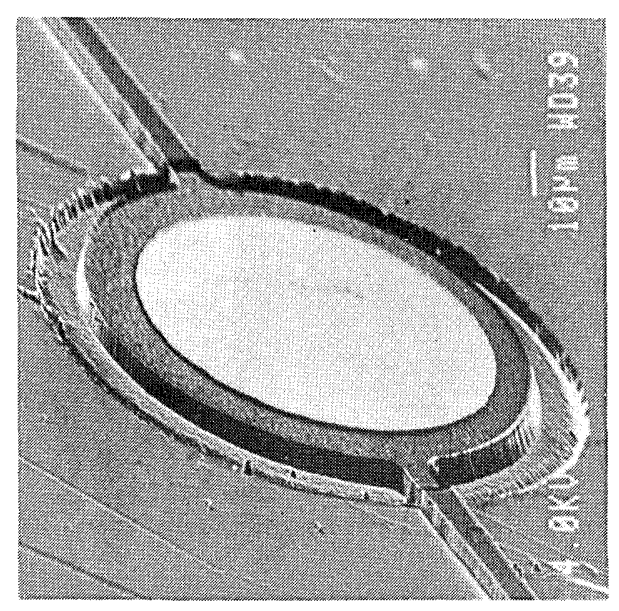

(b)

Fig. 1. (a) Schematic and (b) scanning electron photomicrograph of integrated guided-wave modulator.

proper leads for the application of a bias and was illuminated with a $\mathrm{W}$ source. The transmitted signal was analyzed by a 1-m Jarrell-Ash spectrometer and was recorded by a cooled S1 photomultiplier. Fielddependent data are shown in Fig. 3. The main peak is due to an electron-heavy-hole exciton transition. The shift of the peak to lower energies with bias is due to enhanced electroabsorption in the quantum wells. The quenching of the peak is due to tunneling of carriers out of the respective wells, while the line broadening is due to roughness at the well-barrier interfaces ${ }^{7}$ and possibly to a change in the exciton lifetimes in the presence of the field. Similar data were reported recently by Van Eck et al. ${ }^{8}$ It is seen from our data that a shift of the excitonic peak by nearly $10 \mathrm{meV}$ is obtained at a bias of $15 \mathrm{~V}$.

The intensity-modulation characteristics of the integrated modulator were tested in a device in which the input and output guide lengths were 1.45 and 0.2 $\mathrm{mm}$, respectively. For zero bias applied to the modulator, the device's insertion loss compared with that of a waveguide of the same total length but without the modulator was measured to be $0.9 \mathrm{~dB}$. This is extremely low and indicates that little of the incident light is absorbed or scattered in the intermediate modulating structure. It should also be noted that the guides are designed for single-mode operation, but with our method of coupling light at the input, the reflection losses may be high, and higher-order modes

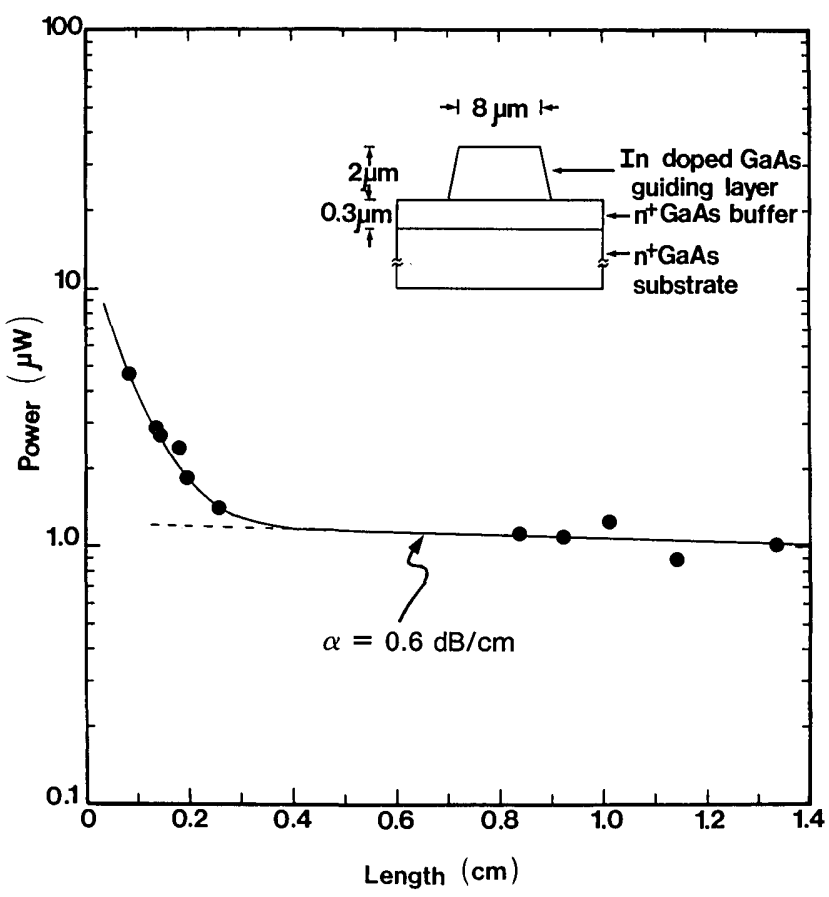

Fig. 2. Output power as a function of $\operatorname{In}_{0.01} \mathrm{Ga}_{0.99} \mathrm{As}$ waveguide length. The guide is $8 \mu \mathrm{m}$ wide and $2 \mu \mathrm{m}$ thick.

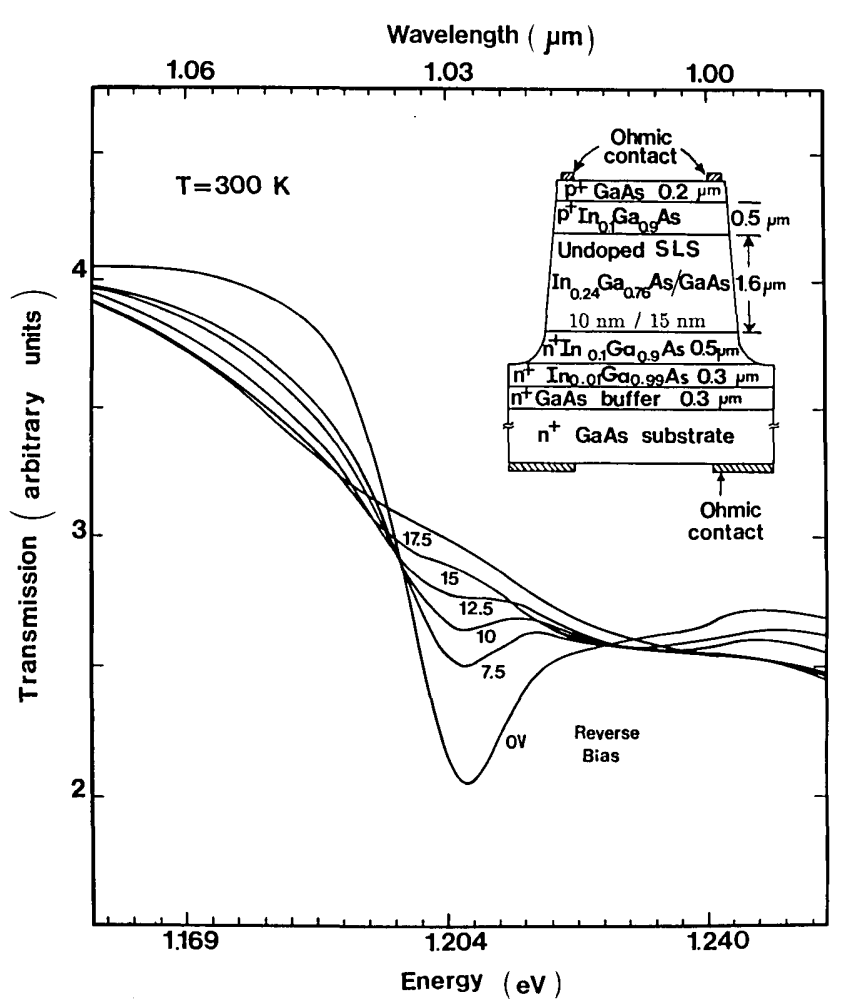

Fig. 3. Field-dependent electroabsorption measured at 300 $\mathrm{K}$ in a $1.6-\mu \mathrm{m}$-thick $\mathrm{In}_{0.24} \mathrm{Ga}_{0.76} \mathrm{As} / \mathrm{GaAs}(10-\mathrm{nm} / 15-\mathrm{nm})$ MQW. 
are launched. Therefore the total loss in the input guide may be high. However, with an integrated laser input or other forms of matched coupling, the total loss of the modulator, inclusive of the guides, can be kept quite small. For an applied bias of $9 \mathrm{~V}$, a $20 \%$ modulation of the incident signal is measured. We attribute this low modulation index to the quality of the $\mathrm{In}_{0.34} \mathrm{Ga}_{0.66} \mathrm{As} / \mathrm{GaAs}$ interfaces in the strained-layer MQW. Other choices of composition and latticematched material can give far superior performance. Furthermore, in the structure used here, the modulator is much larger than the guides, and therefore the light modes are scattered toward the periphery of the device. Therefore the bias is not very effective. A smaller lateral dimension of the modulator will help in this respect.

The impulse responses of the integrated modulators have not been measured directly. However, we have measured the response of as-grown photodiodes made with the same SLS to a pulsed $\mathrm{Al}_{x} \mathrm{Ga}_{1-x} \mathrm{As}$ diode laser. The 100-psec laser pulses are focused to a $50-\mu \mathrm{m}$ spot and are incident normally upon the $125-\mu \mathrm{m}$-diameter diodes. The response is characterized by a FWHM of $150 \mathrm{psec}$ and a convoluted rise time of 115 psec. We have demonstrated here an integrated modulator whose dimensions are much larger than that of the waveguide but that in principle could be rectangular in shape, with the width of the device close to the guide width, as mentioned above. The modulation speed could thereby be greatly enhanced. We are currently in the process of making devices with temporal responses of $\sim 50$ psec or less.

The integrated device demonstrated here can be monolithically integrated with a laser, thus performing as an external modulator. It is also possible, with angled guide facets and proper reflecting surfaces, to transmit light in and out in a perpendicular direction, thereby enabling the device to serve as one element of a spatial light modulator. The guiding regions of the modulator provide the additional advantage of permitting phase shifting of the transmitted signal electro-optically with the application of a bias.

Useful discussions with J. Singh are gratefully acknowledged. The research is being supported by the National Aeronautics and Space Administration under grant NAG-1-555.

\section{References}

1. T. H. Wood, C. A. Burrus, D. A. B. Miller, D. S. Chemla, T. C. Damen, A. C. Gossard, and W. Wiegmann, IEEE J. Quantum Electron. QE-21, 117 (1985).

2. T. H. Wood, C. A. Burrus, R. S. Tucker, J. S. Weiner, D. A. B. Miller, D. S. Chemla, T. C. Damen, A. C. Gossard, and W. Wiegmann, Electron Lett. 21, 693 (1985).

3. P. K. Bhattacharya, S. Dhar, P. R. Berger, and F.-Y. Juang, Appl. Phys. Lett. 49, 470 (1986).

4. U. Das, P. K. Bhattacharya, and S. Dhar, Appl. Phys. Lett. 48, 1507 (1986).

5. H. Inoue, K. Hiruma, K. Ishida, T. Asai, and H. Matsumura, IEEE Trans. Electron Devices ED-32, 2662 (1985).

6. N. Vodjdani, M. Erman, and J. B. Theeten, J. Cryst. Growth 71, 141 (1985).

7. F.-Y. Juang, J. Singh, P. K. Bhattacharya, K. Bajema, and R. Merlin, Appl. Phys. Lett. 48, 1246 (1986).

8. T. E. Van Eck, P. Chu, W. S. C. Chang, and H. H. Wieder, Appl. Phys. Lett. 49, 135 (1986). 